\title{
EL ESCUDO, LA SERPIENTE Y LA MUJER EN ARQUÍLOCO 12 ADRADOS = 5 WEST
}

MARTÍ DURÁN

This paper proposes a new interpretation of fragment 12 Adrados $=5$ West by Archilochus. In my opinion, the hurling of the shield is not to be considered as a sign of disrespect of noble and traditional values or as an indication of cowardice. In fact, since aspis can also mean "viper" and, therefore, "woman" (misogynist conceptions compared the woman to a snake), we put forward a new reading, according to which Archilochus would have been proud of having got rid of what he considered a wicked girl. As the Cologne Epode contains a similar motif, it seems that this kind of sexist scorn was often treated in sympotic contexts, in which we can situate most of Archilochus' poetry.

El fragmento 5 West ha sido interpretado de distintas maneras, pero siempre en una dirección homogénea que no hace más que repetir el contexto en el que nos lo enmarca Plutarco': se dice que Arquíloco presume de su cobardía indicando que, aunque se trata de un defecto, le ha permitido

1 A mi modo de ver, no hay que hacer demasiado caso de los contextos en los que aparece nuestro poema (ni tan siquiera el de Aristófanes) ya que es evidente que el juego de palabras que presenta sólo se comprendió y tuvo sentido en el marco en el que éste fue originariamente ejecutado (para nosotros, un simposio). Por otra parte, al ser posible la interpretación literal, no nos tiene que sorprender que el poema se comprendiera unánimemente como alarde del abandono de las armas. A mi modo de ver, la única clave interpretativa válida es el propio contenido del poema, sólo es posible una interpretación intrínseca.

EMERITA (EM) LXVII 1, 1999, pp. 87-103 
salvar la vida. En este sentido, no le importa haber perdido su escudo ni habérselo cedido a otro (concretamente. a un sayo): pronto se procurará otro, ya que los bienes materiales (no así los inmateriales, como la vida) son reemplazables.El objetivo de estas páginas es analizar el poema desde una triple perspectiva, descifrando lo que a nosotros nos parece una alegoría compleja y, por ello mismo, de gran calidad artística: por una parte, el poema permite la interpretación tradicional; a ésta habría que añadir la derivada de la interpretación de ,spíj como 'serpiente'; finalmente, procediendo de esta segunda posibilidad, tendríamos una tercera: la spíj (en griego, el término es femenino) no sería otra cosa que la mujer (quizá, si se quiere, la propia Neobula ${ }^{2}$. Vamos a ver, sin embargo, en primer lugar, el tenor literal del texto:

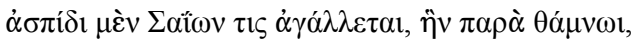

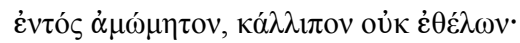
$\alpha$

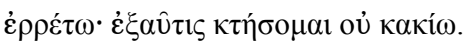

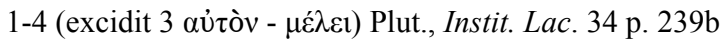

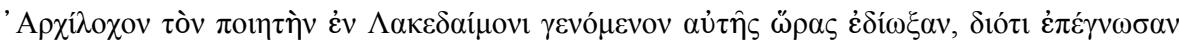

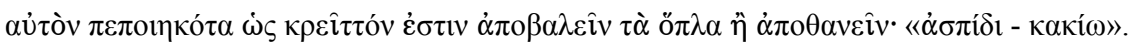

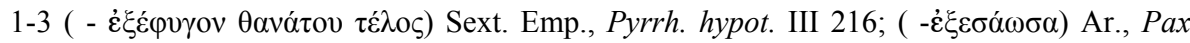

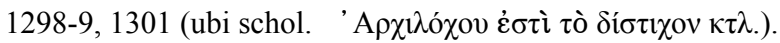

1-2 Strabo X 2.17 p. 457 et XII 3. 20 p. 549 (fort. ex Apollodoro: 244 F 178b); Vita Arati p. 771 Maass.

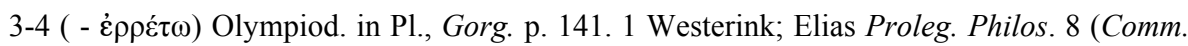
in Arist. Graeca XVIII 22. 21); Ps.-Elias in Porph. Isagogen XII 19, p. 16 Westerink.

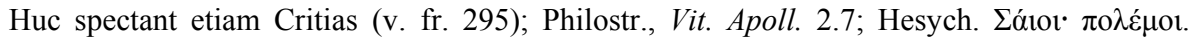

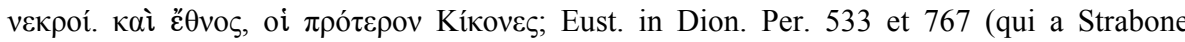
pendet).

2 No es lugar aquí para recordar el noviazgo de Arquíloco con Neobula y la posterior ruptura del mismo. En cambio, sí puede ser interesante reproducir estas palabras de F. Lasserre - A. Bonnard (Arquiloque. Fragments, París, 1968, p. XII) en las que se comenta la terrible reacción que esta ruptura provocó en Arquíloco: «La vengeance d'Archiloque fut terrible et c'est sans doute à cette occasion que se révéla pour la première fois son génie satirique. Sa fureur se déchaîna dans plusieurs épodes: la 1re, contre Lycambe, la 3e, probablement contre Néoboulé donnée pour vieille et débauchée, la 4e, peut-être également dirigée contre Néoboulé peinte en courtisane défraîchie, la 8e, qui semble avoir étalé, de la façon la plus cruelle et la plus grossière, la décrepitude de son ancienne fiancée, devenue obèse putain et dont les hommes, Archiloque y compris, ne veulent plus. Enfin peut-être la 1oe, quoique les indices dont nous disposions soient ici moins clairs». 
carmen integrum; sed cf. Fränkel, Dichtung und Philosophie 2 , p. 152, Wege und Formen 56 n. $3 \| 1 \dot{\alpha} \gamma \varepsilon i ́ \lambda \varepsilon \tau o, \dot{\alpha} v \varepsilon i ́ \lambda \varepsilon \tau o, \dot{\alpha} \varphi \varepsilon i ́ \lambda \alpha \tau o$ et $\tau \grave{\eta} v$ codd. Strabonis (unde etiam $\dot{\alpha} \sigma \pi i ́ \delta \alpha$ p. 549)

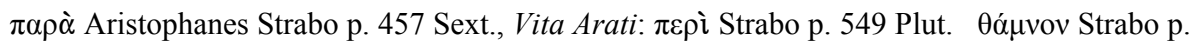

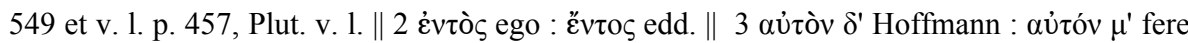

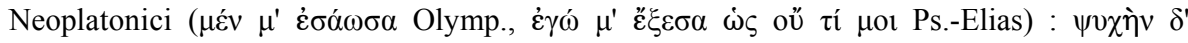

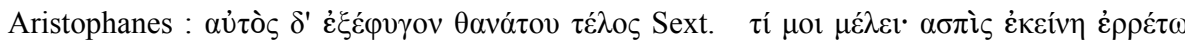

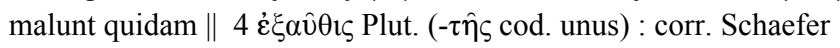

\section{1. 'Aблís como 'escudo': la interpretación tradicional}

La interpretación habitual del fragmento que nos ocupa afirma, como hemos indicado, que Arquíloco abandonó su escudo (lo cual equivale a decir, metonímicamente, que abandonó la guerra) pero que, a cambio, salvó su vida. Esta actitud de Arquíloco se conoce técnicamente como ṕ $\psi \alpha \sigma \pi i ́ \alpha$ y en la Atenas clásica estaba incluso condenada legalmente (mediante una $\delta \varepsilon ı \lambda i ́ \alpha \varsigma$ $\gamma \rho \alpha \varphi \eta ́$, datable de la época de Solón; por otra parte, el pí $\psi \alpha \sigma \pi ı \varsigma$ se convertía en $\left.\alpha^{\prime} \tau \mu \mathrm{o}^{3}\right)$. Dos autores que han defendido recientemente esta interpretación son Di Benedetto ${ }^{4}$ y Schwertfeger ${ }^{5}$. Este último se podría considerar un representante prototípico de esta corriente interpretativa tradicional: en el fondo, no hace más que utilizar el poema de Arquíloco como pretexto para analizar la j̇ $\psi \alpha \alpha \sigma \operatorname{tí}_{\alpha}$ en las distintas épocas de la civilización griega como concepto jurídico y social. Se desprende de las

3 Aisch. III 175 = E. Ruschenbusch, $\Sigma O \Lambda \Omega N O \Sigma$ NOMOI, Wiesbaden, 1966, F. 110.

4 V. Di Benedetto, «Archil. fr. 5 W», Eikasmos 2, 1991, pp. 13-27.

5 T. Schwertfeger, «Der Schild des Archilochos», Chiron 12, 1982, pp. 253-280. Otras

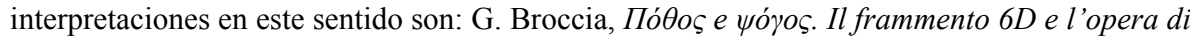
Archiloco, Roma, 1959; W. Donlan, «The Tradition of Anti-Aristocratic Thought in Early Greek Poetry», Historia 22, 1973, p. 146; H. Fränkel, Dichtung und Philosophie des frühen

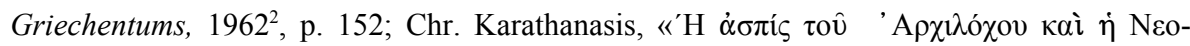

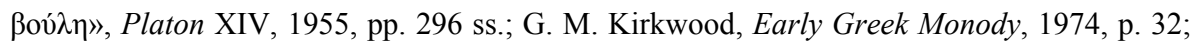
A. J. Podlecki, «Three Greek Soldier-Poets: Archilochus, Alcaeus, Solon», ClW 63, 1969, pp. 74ss.; B. Snell, Die Entdeckung des Geistes $1975^{4}$, pp. 61ss. Véase también C. M. Bowra, Greek Lyric Poetry, 1962², pp. 138ss.; K. J. Dover, «The Poetry of Archilochus», Entretiens sur l'antiquité classique (Fondation Hardt) 10: Archiloque, 1964, pp. 196ss.; C. Gallavotti, «Archiloco», PP 4, 1949, pp. 136ss.; R. Harder, «Zwei Zeilen des Archilochos» Hermes 80, 1952, pp. 381ss.; W. Jäger, Paideia 1, 1934, p. 165; H. D. Rankin, Archilochus of Paros, 1977, pp. 42 y sigs.; H. D. Rankin, «MOIXO $\Sigma, \Lambda$ AГNO $\Sigma$ KAI YBPI $\Sigma T H \Sigma$ : Critias and His Judgement of Archilochus», Grazer Beiträge 3, 1975, p. 324, n. 4; B. Seidensticker, «Archilochus and Odysseus», GRBS 19, 1978, pp. 20ss.; M. Treu, Archilochos, 1959, p. 192. 
consideraciones de Schwertfeger que la jıł $\alpha \sigma \pi i ́ \alpha$ experimentó una evolución a lo largo de las épocas. Así, mientras en época clásica (tanto en Atenas ${ }^{6}$ como en Esparta ${ }^{7}$ ) el ṕí $\alpha \alpha \sigma \pi \varsigma$ era considerado como un desertor perseguible penalmente (lo hemos visto) en la época homérica y -según Schwertfeger- también en la arquiloquea, este mismo personaje actuaba por motivos meramente personales (salvar su vida) y no era mal visto por la sociedad: en realidad, el propio Alceo comenta el abandono de su escudo, al parecer, ante sus propios compañeros de banquete $^{8}$. Según Schwertfeger, esta evolución en la consideración de la ṕ $\psi \alpha \sigma \pi i ́ \alpha$ se debe al cambio de técnicas defensivas: efectivamente, en la época clásica la formación hoplítica convertía en extremadamente perjudicial para los demás la deserción de uno solo 9 . También por ello es a partir de la época clásica cuando se empieza a valorar negativamente el poema de Arquíloco $^{10}$. Si retrocedemos ahora a la época homérica - más cercana a Arquíloco - vemos, por una parte, que se desconoce la $\dot{\rho} \iota \psi \alpha \sigma \pi i ́ \alpha$ como tal (hay abandono de armas no especificadas y no, concretamente, del escudo) y, por otra, que este abandono no implica una deserción punible, sino que está al servicio de las necesidades prácticas ante

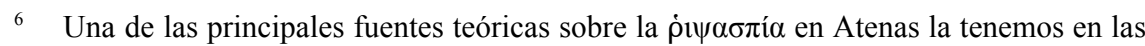
Leyes de Platón (943A-945B). Cf. también Lisias 10. 9 y las distintas alusiones en Aristófanes (la más importante en Pax 1295-1304).

7 Para Esparta, cf. Tirteo fr. 10-12 West, Diod. 12, 62, 4 y algunos Apophthegmata Laconica de Plutarco (239B, 220A, 241F, etc.).

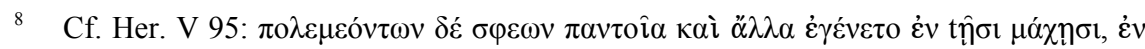

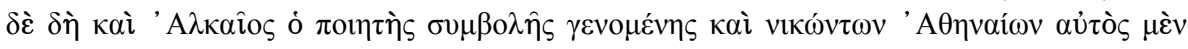

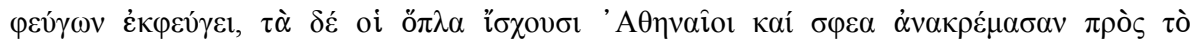

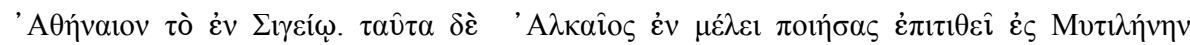

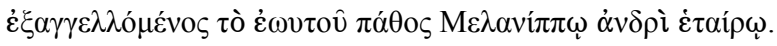

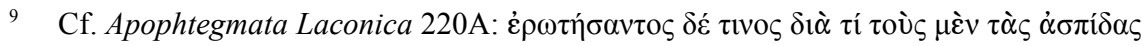

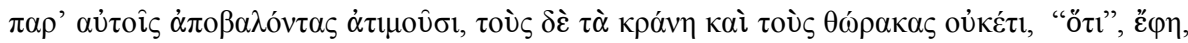

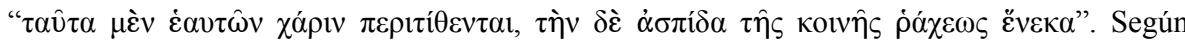
Schwertfeger (ob. cit., p. 276) «dies ist die pointierteste Definition der Funktion des Schildes. Er dient nicht wie die sonstigen Waffen dem persönlichen Schutz, sondern dem Schutz der Schlachtreihe, die ja eine Formation Schild an Schild darstellt».

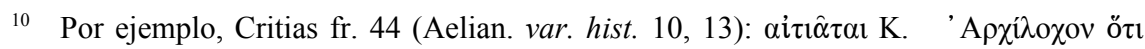

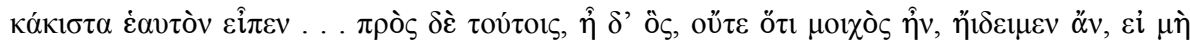

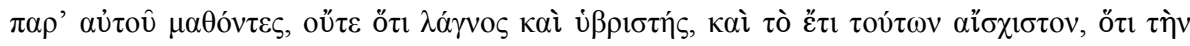

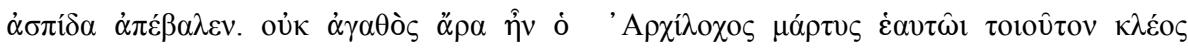

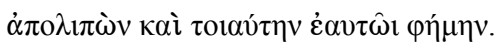


las circunstancias de la guerra ${ }^{11}$. En palabras de Schwertfeger, «das Epos kennt also die $\dot{\rho} \psi \psi \alpha \sigma \pi i ́ \alpha$ als eine Form der Kapitulation, nicht aber der Flucht in der Schlacht ${ }^{12} \gg$. Es así como se deben analizar algunos pasajes homéricos, como por ejemplo Il. VIII 93-98, Il. VIII 139-171, Il. XI 408410, Il. XIV 65 ss., Il. XXI 76 ss., Il. XXII 111-125, Od. XIV 276-279, etc ${ }^{13}$. A nuestro modo de ver, esta serie de reflexiones son muy válidas y sin duda hay que tenerlas en cuenta para interpretar el poema de Arquíloco que, consciente de lo que probablemente era un motivo literario ${ }^{14}$, juega con ellas y las utiliza para provocar la hilaridad utilizando las posibilidades de este primer nivel de lectura. Sin embargo, nos parece que quedarse en este nivel supone no sacar todo el provecho del poema ni, sobre todo, explica algunos de sus puntos. Un primer aspecto que no queda bien explicado con esta

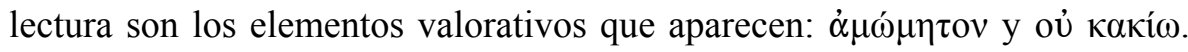
Evidentemente, estos adjetivos se pueden aplicar a un escudo, pero parece extraño que se califique de 'irreprochable' o 'no más malo' a un objeto, ya que estas cualidades parecen adecuarse mejor a una persona ${ }^{15}$. Por otra parte,

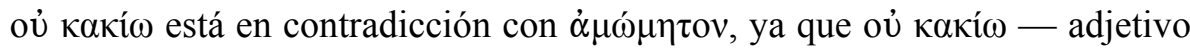
en grado comparativo — pone en relación dos realidades negativas, mientras

11 Según H. D. Rankin, «Archilochus fr. 6 D (13 L/B; 8 T», LF XCVIII, 1975, pp. 193198, declarando con simplicidad que ha lanzado su escudo para salvar su vida, Arquíloco no ridiculiza la ética de la nobleza homérica, sino que simplemente demuestra que ya no tiene razón de ser en una época de batallas en fila. En efecto, si el heroismo daba excelentes resultados en el combate cuerpo a cuerpo, un hoplita era en cambio mucho más útil preparándose para un nuevo combate que persistiendo en una fila descompuesta.

12 Ob. cit., p. 261.

13 Según Schwertfeger (ob. cit., p. 257) «die Flucht ist für die epischen Helden also ein differenziert zu sehendes Vorkommnis. Sie wird als ehrlos geschmäht oder als gottgesandt hingenommen. Auf der anderen Seite aber kann die Flucht ganz nüchtern erwogen werden (Agamemnon), sie kann aus wohlerwogenen Gründen nützlich sein (Nestor), oder sie kann einfach stattfinden (Odysseus). In jedem Fall trägt sie nicht mehr als den aktuellen Tadel eines Mitkämpfers ein, d. h. sie ist für den Betreffenden folgenlos und entehrt ihn nicht».

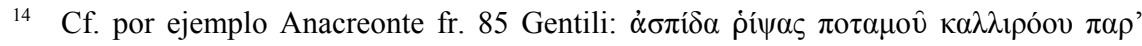
ő $\chi \propto \alpha \varsigma$ (por cierto, no me extrañaría que en este verso hubiera también alguna alusión erótica) y Hor. Od. II 7 9-12: tecum Philippos et celerem fugam / sensi relicta non bene parmula, / cum fracta uirtus, et minaces / turpe solum tetigere mento.

15 ' A $\mu \omega ́ \mu \eta \tau$, 
que $\dot{\alpha} \mu \omega ́ \mu \eta \tau$ ov se refiere únicamente a cualidades positivas ${ }^{16}$. En estas circunstancias, parece extraño que Arquíloco se lamente tanto de haber abandonado un escudo que, al fin y al cabo, era каки́.

Otro elemento que no queda especificado con esta interpretación es el étnico $\Sigma \alpha i ̂ i \omega v$. Los sayos son una tribu tracia vecina a Abdera. Hesiquio los

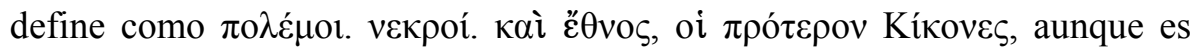
evidente que el primer elemento de esta definición procede del propio poema de Arquíloco, por lo que no tiene, a mi modo de ver, demasiado valor (como explica Hiller von Gaertringen ${ }^{17}$, es probable que los tracios tuvieran conflictos con los habitantes de Paros a raíz de la colonización de Tasos). A

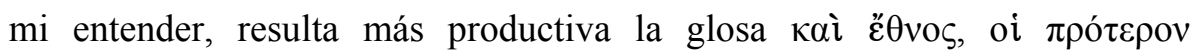
Kíkovєc. Es sabido que los tracios eran considerados como grandes borrachos (era proverbial el "beber como un tracio"18); en este sentido, los Kíkovę son no sólo tracios sino, además, habitantes de la ciudad de Ismaro, productora de uno de los vinos más famosos de la Antigüedad, alabado por

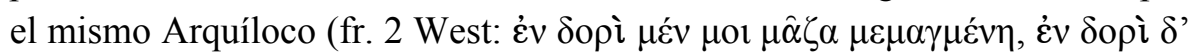

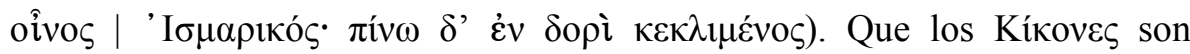
habitantes de la ciudad de Ismaro o de sus inmediaciones lo sabemos por numerosos pasajes, entre los cuales podemos destacar los siguientes:

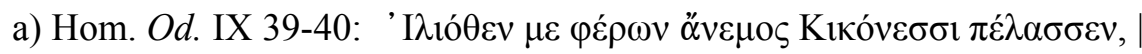

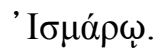

b) Schol. Hom. Od. IX vं

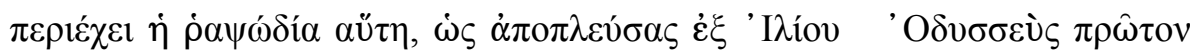

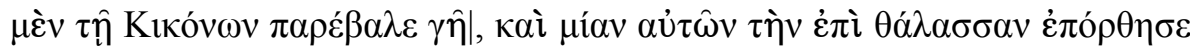

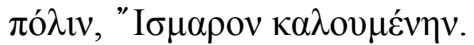

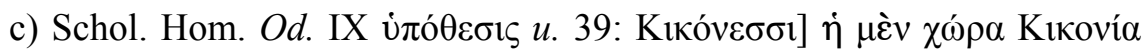

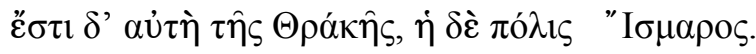

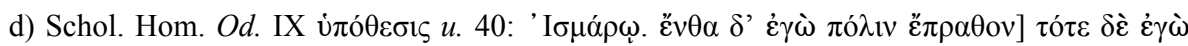

16 Por cierto: en el Papiro de Colonia (fragmento 196a West), 1. 7, encontramos la

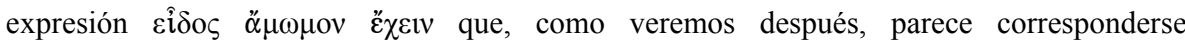

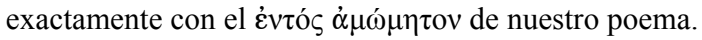

17 «Noch einmal das Archilochosdenkmal von Paros», $N G G$ (N.F.) 1, 1934, pp. 41-58.

18 Cf. por ejemplo Horacio (Carm. I 27 1-4): Natis in usum laetitiae scyphis / pugnare Thracum est: tollite barbarum / morem uerecundumque Bacchum / sanguineis prohibete rixis. 


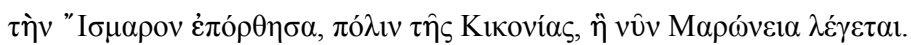

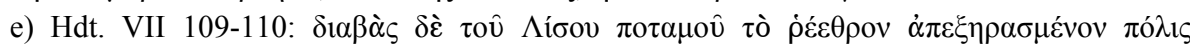

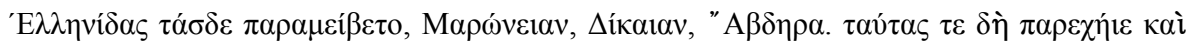

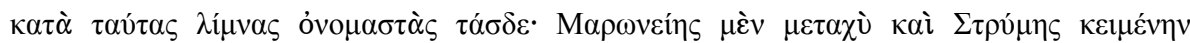

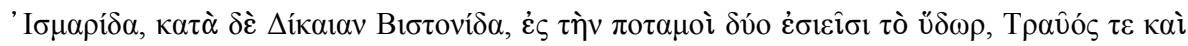

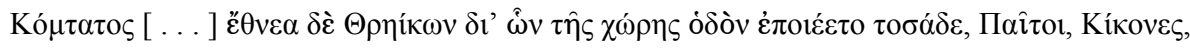

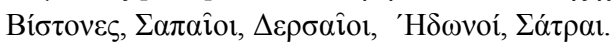

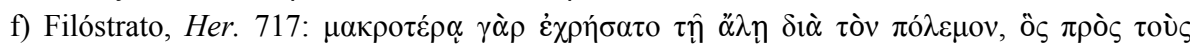

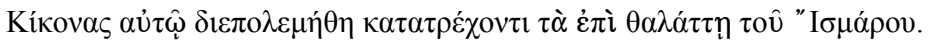

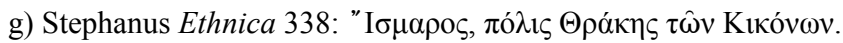

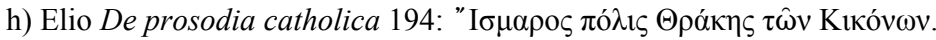

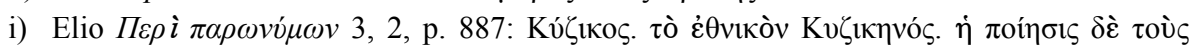

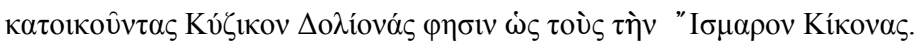

Desde esta perspectiva, "sayo" equivaldría a "borracho" o "inclinado a la bebida". Veremos después la transcendencia de esta palabra, en un contexto como el que nos ocupa.

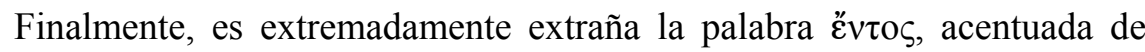
esta manera y entendida como "arma". Como consta en el $L S J$ s.u., el poema

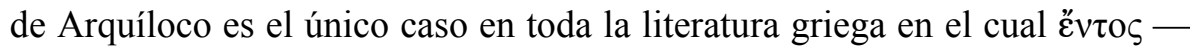
un término de uso frecuentísimo - aparece en singular y no en plural ( $\varepsilon^{\prime} v \tau \varepsilon \alpha$ ), ya que esta palabra pertenece a la categoría de los denominados pluralia tantum. Por otra parte, en la interpretación tradicional del poema hay una sintaxis posible, pero un poco alterada, en la oración de relativo: complemento circunstancial, aposición a un elemento que ha aparecido a bastante distancia, verbo y participio predicativo. A mi modo de ver, partiendo de otra estructura (complemento circunstancial, adverbio, predicativo, verbo y participio apositivo, es decir: "a la que en un matorral, aun siendo irreprochable en su interior, dejé y no quise") resulta posible y

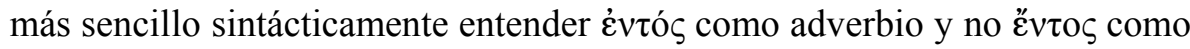
substantivo (aunque, recordemos nuestra insistencia en la doble lectura, sin duda la homofonía de las palabras y el adjetivo de dos terminaciones - y no de tres - $\dot{\alpha} \mu \omega ́ \mu \eta \tau$ ov responden a un afán evidente de dilogía). De esta manera, Arquíloco afirmaría que, a pesar de la belleza de la mujer (o bien:

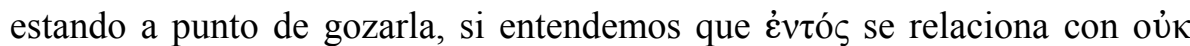
$\left.\dot{\varepsilon} \theta \varepsilon ́ \lambda \omega v^{19}\right)$ la habría rechazado por ser una "víbora", consolándose con la

19 Esta expresión puede muy bien referirse a la falta de deseo amoroso. Cf., en este 
certeza de encontrar pronto a otra no peor. Pero veamos a continuación a partir de qué elementos se puede considerar que Arquíloco se refiere a una "víbora".

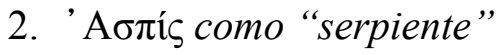

El término $\dot{\alpha} \sigma \pi i ́ \varsigma$ (y muchos de sus derivados) significa en griego, además de "escudo", "serpiente". El DGE (s.u. $\dot{\alpha} \sigma \pi i ́ \varsigma)$ da dos posibilidades de traducción en este sentido: "áspid de Egipto o de Cleopatra, Coluber haié" (Hdt. 4. 191, Nic. Th. 158, Ph. 2. 570, Ael. NA 10. 31, D. C. 51. 11. 2 y Ar.

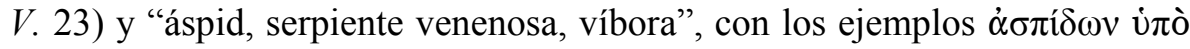

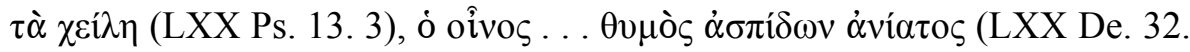
33) y $\omega \grave{\alpha} \dot{\alpha} \sigma \pi i \delta \omega v ~ \varepsilon ̋ \rho \rho \eta \xi \alpha v$ (LXX Is. 59. 5).

Si tenemos en cuenta este significado, nuestro poema ofrece un contenido hasta ahora insospechado. Arquíloco se alegraría de haber dejado apartada en un matorral a una serpiente que ha estado a punto de morderle $\mathrm{y}$, en consecuencia (tengamos en cuenta las características mortíferas del áspid), de matarle. Desde esta perspectiva, se entienden bien dos términos que de otra forma resultarían difícilmente comprensibles: $\theta \alpha \dot{\mu} \mu \nu o \varsigma$ у $\dot{\varepsilon} \xi \varepsilon \sigma \alpha ́ \omega \sigma \alpha$. La aparición del matorral (aparte del significado erótico que después le veremos) es muy significativa en conexión con el término $\dot{\alpha} \sigma \pi i ́ \zeta$, ya que las serpientes se refugian en matorrales. Por otra parte, la expresión "salvar la vida" se entiende bien si uno ha estado a punto de ser mordido por una serpiente venenosa, que ha acabado abandonando entre los hierbajos.

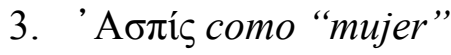

A partir del significado de "serpiente", el término $\dot{\alpha} \sigma \pi i ́ \varsigma$ pasa a designar en griego a la mujer o, más bien dicho, a la "mala mujer". Este significado (aunque tiene antecedentes, recordemos el ejemplo del Génesis) no consta en

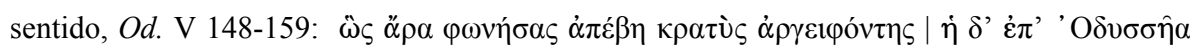

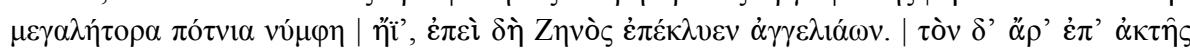

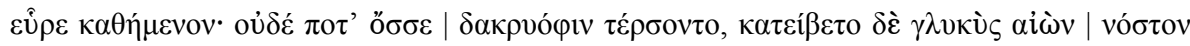

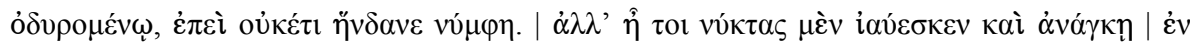

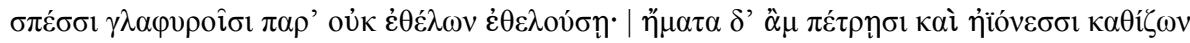

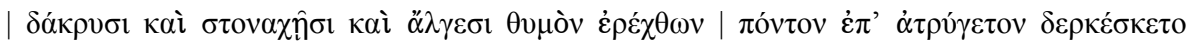

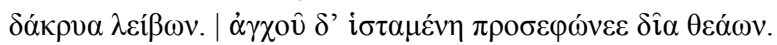


nuestros léxicos ${ }^{20}$ pero, a mi entender, puede considerarse implícito en una gran cantidad de pasajes, de los que ahora señalaré sólo unos cuantos. Clitemnestra, paradigma de la mujer malvada que participa como autora o cómplice en la muerte de su marido Agamenón, es calificada por su hijo

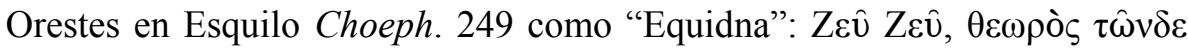

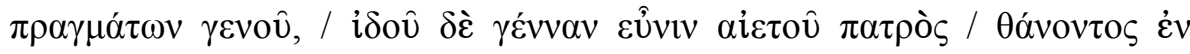

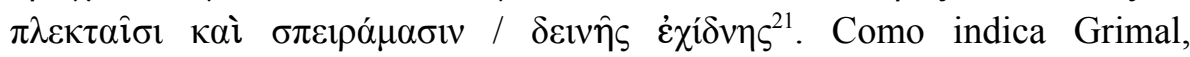
«Equidna es la 'Víbora', monstruo con cuerpo de mujer, terminado por una cola de serpiente en lugar de piernas $\rangle^{22}$. Recordemos que, precisamente por esta cualidad, Heródoto (IV 9) califica a la Equidna como $\mu \varepsilon 1 \xi o \pi \alpha ́ \rho \theta \varepsilon v o \varsigma$

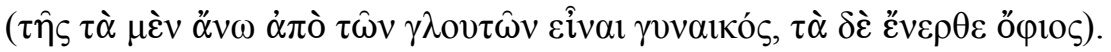

Dión de Prusa, en su quinto discurso, nos habla de unas mujeres libias con características ofídicas. Según Dión, estas mujeres atraían sexualmente con su postura a los caminantes, mostrándoles sus pechos (ya que en su parte superior eran mujeres, y sólo a partir de medio cuerpo se convertían en serpientes). Una vez los habían atraído, los poseían (el desenfreno sexual de las mujeres-serpiente es un elemento recurrente) y los devoraban.

Sin embargo, la figura mítica que, en sus orígenes, podemos pensar que tenía una relación más estrecha con la serpiente es Helena. Con variantes de detalle, el mito nos cuenta que los dos pilotos (Canopo o Faros) que la condujeron hacia Egipto murieron por la mordedura de una serpiente. En las diversas narraciones, que nos han sido conservadas en relatos tardíos y

20 En cambio, sí ha sido analizado en distintos artículos. Citemos, entre los más emblemáticos, J. A. Álvarez Osés, «La mujer y la serpiente», Caesaraugusta 21-22, 1964, pp. 49-74 y M. Sancassano, «Il mistero del serpente: retrospettiva di studi e interpretazioni moderne», Athenaeum. Studi di Letteratura e Storia dell'Antichità, vol. 85. 2, 1997, pp. 355390. Álvarez Ossorio (ob. cit., pp. 56-58) estudia distintas conexiones entre la mujer y la serpiente, como las figurillas de época prehelénica de la isla de Creta, las diosas de la fecundidad Ártemis arcadia y Perséfone, las representaciones de Atenea con serpientes en las Panateneas, la Medusa Gorgona, etc. Según este autor (ob. cit., p. 51) «la relación entre la mujer y la serpiente adopta casi siempre una forma sexual».

21 En realidad, como comenta A. F. Garvie (Aeschylus. Choephori, Oxford, 1988, p. 107) «Clytemnestra is a snake at $\mathrm{Ag} .1233$, Cho. 994, 1047, and Orestes becomes a snake at 527 ff., 549, 928».

22 P. Grimal, Diccionario de Mitología griega y romana, trad. esp. F. Payarols, Barcelona, 1986, s. u. "Equidna". 
parciales o alusiones, la atracción sexual parece haberse mezclado oscuramente con la muerte. Aunque no se dice explícitamente en ningún lugar que Helena fuera una serpiente, es significativo que los dos pilotos fueran atraídos por Helena y encontraran la muerte víctimas de una serpiente. En este sentido, hay que recordar que Helena había estado relacionada con el árbol ${ }^{23}$ y que, según Nicandro, se contaba que de las lágrimas de Helena, afligida por la muerte de Canopo, nació el helenion, una hierba que curaba las mordeduras de serpiente. También hay quien dice que, en esta misma segunda estancia egipcia, confiada Helena a la protección de Tonis, la mujer de éste, al ver que su marido sentía atracción por Helena, la desterró a la isla de Faros, que estaba llena de serpientes y le dio, como antídoto contra las mordeduras, la hierba que hemos comentado. La literatura cristiana contiene, como era de esperar, abundantes referencias a la consideración de la mujer como serpiente. Aunque esta identificación tiene sin duda raíces semíticas (estudiadas por Phillips ${ }^{24}$ ) ha sido un mérito de Gilabert ${ }^{25}$ distinguir en algunos de estos textos misóginos importantísimas influencias griegas (por ejemplo, del Fedro de Platón), de manera que podemos usar algunos textos misóginos cristianos como reflejo de las concepciones griegas que nos interesa ahora analizar. Entre estos textos, es especialmente significativo uno de Filón de Alexandría ${ }^{26}$. He aquí cómo

23 Pausanias (III 19 10) habla de un culto dorio a Helena dendritis. Cf. W. Mannhardt, Wald- und Feldkulte II, Antike Wald- und Feldkulte, Berlin, 1887 (reimpr. 1963).

24 J. A. Phillips, Eva. La historia de una idea, México, 1984, cap. IV. Recordemos que "Javvah" -Eva- parece derivar de las palabras árabes o arameas que significan 'serpiente': en arameo, por ejemplo, 'serpiente' se dice 'hiwja'.

25 P. Gilabert, «'... Però la dona ho esguerrà tot'. El De opificio mundi de Filó d'Alexandria (LIII-LXI), o els fonaments grecs d'una fita en la història de la misogínia occidental», Anuari de Filologia, XIII D, 1990, pp. 55-84. Véase también, del mismo autor, «El De opificio mundi de Filó d'Alexandria, o les lleis de l'al·legorisme clàssic al servei de la teologia pedagògica», Universitas Tarraconensis XI, 1987, pp. 107-122.

26 De opificio mundi LVI-LVII (157-161). Otro texto importante en el mismo sentido es

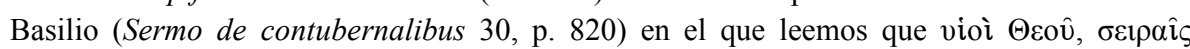

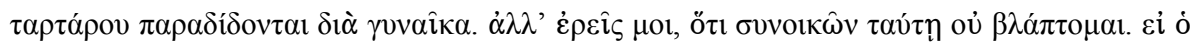

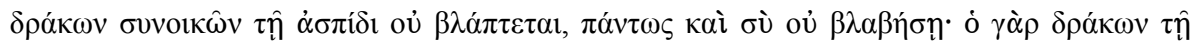

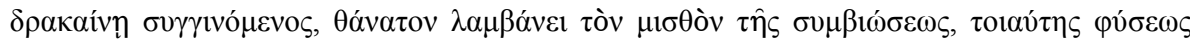

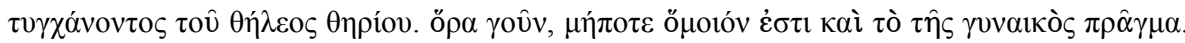
Obsérvese que la mujer es calificada exactamente de $\dot{\alpha} \sigma \pi i \varsigma$, es decir, el término que nos 
comenta Filón el relato bíblico del pecado original: «todo esto no son ficciones propias del mito (oủ $\mu v ́ \theta$ ov $\pi \lambda \alpha \dot{\sigma} \sigma \mu \alpha \tau \alpha$ ), con las que se complacen el género poético y sofístico, sino manifestación de figuras que nos exhorta a la interpretación alegórica ( $\dot{\varepsilon} \pi$ ' $\dot{\alpha} \lambda \lambda \eta \gamma$ píav) para dar razón de los significados escondidos (i்ovol $\hat{\omega} v)$. Diremos, pues, con toda conveniencia, por seguir una conjetura verosímil ( $\varepsilon i \kappa o ́ \tau \imath ~ \sigma \tau o \chi \alpha \sigma \mu \hat{\omega})$ que la repetida

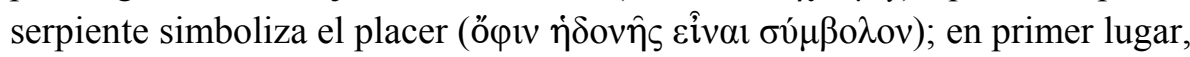

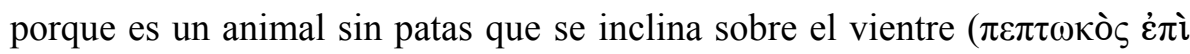
$\gamma \alpha \sigma \tau \dot{\varepsilon} \rho \alpha)$; en segundo lugar, porque se nutre de migas de tierra ( $\gamma \hat{\eta} \varsigma \beta \omega ́ \lambda$ ors); $\mathrm{y}$, en tercer lugar, porque lanza su veneno por los dientes, arma natural con la que mata a los que muerde. Aquél que desea el placer (ó $\varphi \imath \lambda \eta ́ \delta o v o \varsigma)$ no deja de participar de ninguno de los rasgos citados. Dado que se encuentra

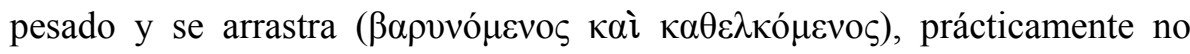

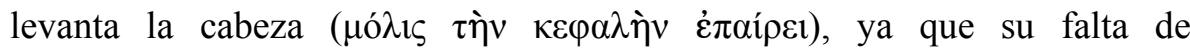

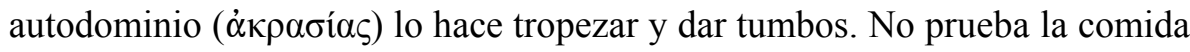

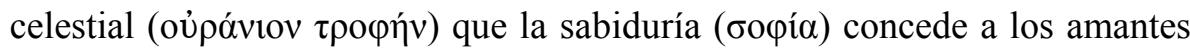

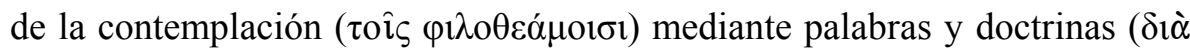

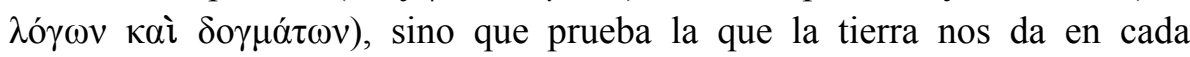

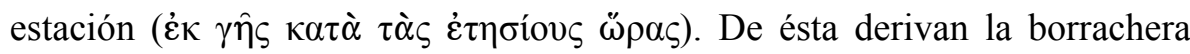

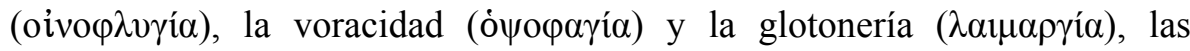

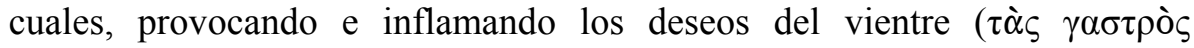
$\dot{\varepsilon} \pi \imath \theta v \mu i ́ \alpha \varsigma)$, hacen crecer y estallar el aguijón del bajo vientre ( $\tau \grave{\alpha} \varsigma \gamma \alpha \sigma \tau \rho \grave{\varsigma}$ $\dot{\varepsilon} \pi\left(\theta v \mu i_{\alpha} \alpha\right)$. Disfruta entonces del trabajo de panaderos y cocineros $\mathrm{y}$, girando la cabeza, intenta captar el olor de las comidas. En el caso de que vea una mesa llena, se lanza a ella con ansia de tragárselo todo, una cosa tras la otra, teniendo como objetivo no ya saciarse, sino agotarlo todo; es por eso que lleva, no menos que la serpiente, el veneno entre los dientes. Ellas son, efectivamente, las servidoras y criadas de la glotonería, ya que lo trocean y hacen todo añicos para poderlo comer. En primer lugar, pasan las comidas a la lengua para saborearlas y, después, a la garganta. Por otra parte, la falta de medida en la comida es mortífera y venenosa por naturaleza, ya que la digestión no se puede llevar a cabo si se introducen otros alimentos antes de evacuar los primeros. Se dice también que la serpiente emitía voces humanas, precisamente porque el placer (i்oví) se aprovecha de 
innumerables defensores para su custodia y protección, los cuales se atreven a afirmar que su poder se extiende a todos, pequeños y mayores, sin excepción. Y, ciertamente, los primeros encuentros entre hombre y mujer

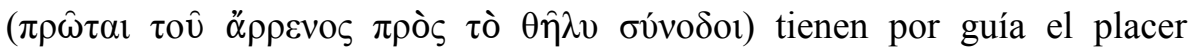
(ं்oví)». A mi entender, es evidente — a pesar de los titubeos de Gilabert ${ }^{27}$ - que aquí la mujer se identifica con la serpiente, ya que el "placer" procede por antonomasia de ésta. Finalmente, dos sentencias de Menandro indicarían que la comparación de la mujer con una $\dot{\alpha} \sigma \pi$ íc formaba parte del

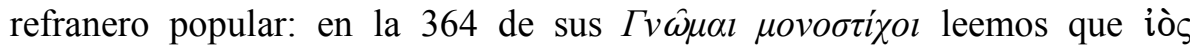

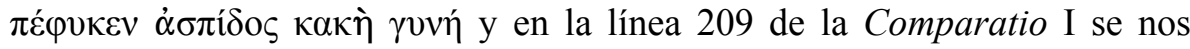

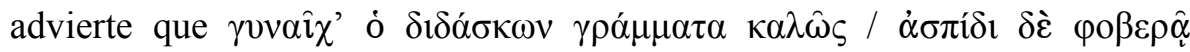

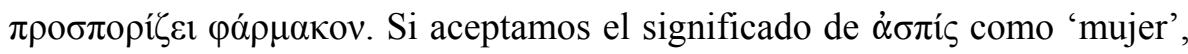
el poema que nos ocupa vendría a decir lo siguiente: la mujer con quien Arquíloco había estado ha pasado a relacionarse por otra persona (un sayo). Arquíloco, sin embargo, no se muestra en absoluto preocupado: él mismo la ha abandonado en un momento en que le era posible gozar de ella. Y lo ha hecho por considerarla, aunque bella, una "víbora", una mala mujer. Por ello, no cree que esta pérdida constituya ninguna desgracia ( $\dot{\varepsilon} \rho \rho \varepsilon ́ \tau \omega)$ y se ve capaz de procurarse rápidamente otra mujer mejor. Algunos de los elementos del poema adquieren su verdadero significado desde esta tercera posibilidad interpretativa. Vamos a ver los más significativos:

27 Según Gilabert (ob. cit., p. 70): «sens dubte cap home sensual no negaria les incomoditats de la borratxera, de l'empatx, etc., però, per què no confessar igualment els perills de la 'meula i golafreria espirituals'? Filó en negaria la possibilitat, naturalment, i, tanmateix, aplicant-hi una mica de rigor aristotèlic, els seus raciocinis, podrien esdevenir veritablement brutals: La serp és símbol del plaer; l'amant del plaer és com una serp; l'home acompanyat de dona tasta el plaer; ergo l'home casat amb dona és una serp. Segona versió també possible- encara més marcadament misògina: La serp és símbol del plaer; l'amant del plaer és com una serp; les primeres relacions home-dona tenen per guia el plaer; ergo, l'home casat amb dona estima una serp. Gosaria dir, i sense moure'ns de l'àmbit religiós, que entre aquestes relacions mascle-femella, segons Filó tan hipogàstriques, i aquelles Déu-home tan celestials, hi deu haver la gran distància -segurament abismal- que entre el celibat entès com a simple 'estratègia' no exempta de sacrifici per a la consecució d'uns fins -no gens obsés, per tant-, i aquell altre celibat propi dels qui sempre han capit la dona com a gran temptació, i el matrimoni com a institució de la classe vulgar, totalment aliena a la 'puresa' dels 'rectors'». 
a) El matorral ( $\theta \dot{\alpha} \mu v o \varsigma)$ : el matorral representa el lugar para hacer el amor y equivale en este sentido a los "jardines" o a las "flores" del fr. 196a West (el famoso Epodo de Colonia) ${ }^{28}$. Como se ve en este poema, la presencia del jardín ${ }^{29}$ (en el caso despectivo del fr. 5 West, en

28 Los versos más significativos para lo que nos interesa demostrar aquí son los

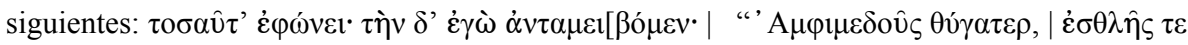

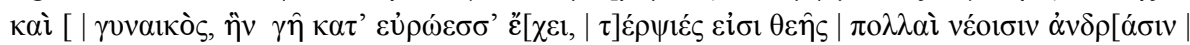

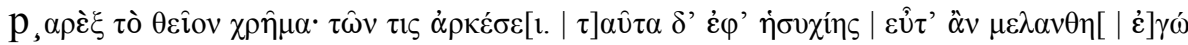

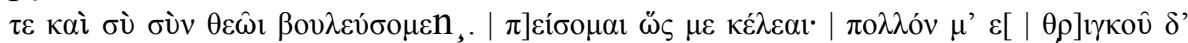

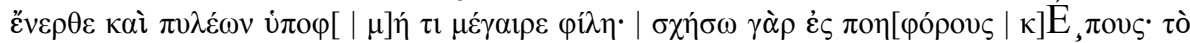

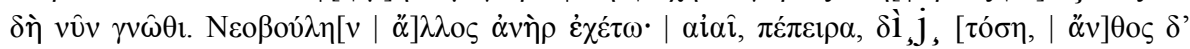

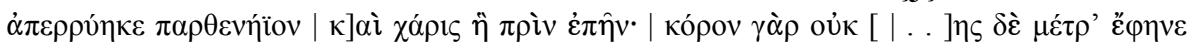

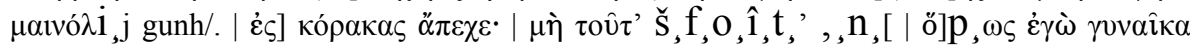

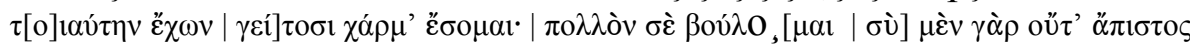

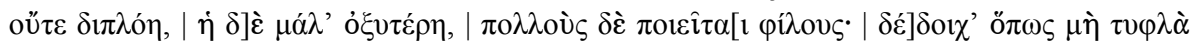

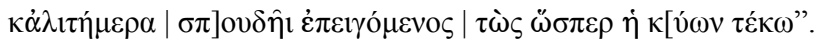

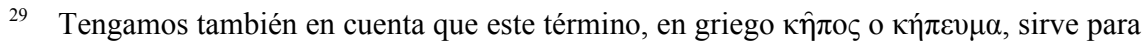
referirse al sexo femenino (cf. Aristófanes Av. 159 y 1101). Carles Garriga ha hablado, en este sentido, de «il prato delle donne» (cf. la conferencia «Il prato delle donne», pronunciada en Trieste el año 1997, todavía inédita, y de la cual me autoriza amablemente a citar los siguientes párrafos): «Il prato, o il giardino, possono essere un greco, come in altre lingue, metafore del corpo della donna o, più concretamente, del sesso femminile. Ne risultano espressioni di tipo osceno, di frequenza variabile secondo il genere letterario e l'epoca: più normali in contesti comici e più abituali in testi tardi. Tra questi troviamo un passo di Diogene Laerzio (II 116) in cui un personaggio di nome Theodoros, rispondendo ad un discepolo di Euclide che dichiarava che 'Atena non è un dio (théos) ma una dea (theá), perché soltanto i maschi possono appellarsi dei', disse: 'Come fa Stilpone a saperlo?' Le ha forse sollevato le vesti e le ha visto il giardino?' - Vi sono, ovviamente, corpi femminili, donne con corpi che si tolgono le vesti e metafore del corpo. Aristeneto (Ep. 1. 3, 26) parla, ad esempio, di un erotikós parádeisos, con una cortigiana di nome Leimóne, e ad Ep. 2. 1 spiega la ragione per cui la donna è come un prato. Tuttavia Aristeneto ed anche Alcifrone (IV, 13 = fr. 6 ed. Loeb), che ne è la fonte, scrivono con la convinzione che l'unica maniera possibile di fruire della natura è utilizzandola dal di 'fuori', abolendo l'idea dello spazio agricolo come scenario idillico; coerentemente, anche la donna è un corpo e nient'altro che un corpo: non è stranno che Leimone sia una prostituta né che questo tipo di opere abbiano meno esperienza artistica genuina che ekfráseis centenarie che finiscono col servire di risposta alle sollecitazioni di progymnásmata. - Anche la commedia, com'è noto, si compiace di presentare, con le metafore corrispondenti, il corpo femminile, e anche quello maschile. Tuttavia il genere comico accoglie più facilmente di altri la lingua viva e, per di più, è teatro: i corpi sono necessariamente presenti nell'opera. Nonostante ciò, la presenza dei corpi e l'uso regolare della lingua quotidiana sono, senza per questo essere aboliti, superati per accedere all'utopia comica, disegnata secondo il modelo di una festa di tipo arcaico, dove 
el que se invierten los términos, del "matorral") implica la inmediatez del acto sexual. Desde esta perspectiva, el significado de nuestro poema está claro: a pesar de tener la oportunidad de gozar de una mujer, Arquíloco la ha despreciado y la ha abandonado.

b) "Me procuraré otra no peor": el significado de esta expresión se entiende bien a partir de la misoginia griega y, esencialmente, de los poetas arcaicos. La mujer nunca puede ser buena: por ello, una mujer mejor que la abandonada sólo puede ser "no peor". Para entender esta idea, puede ser interesante repasar la concepción hesiódica de la mujer ${ }^{30} \mathrm{o}$ bien, en un ámbito más próximo, la de algunos poetas arcaicos: Semónides, por ejemplo, después de hacer un lamentable catálogo de las distintas posibilidades de mujeres - a cual peor - escribe ${ }^{31}$ que

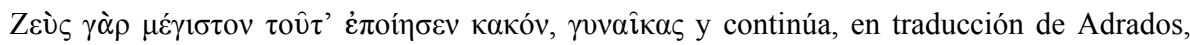
«cuando más satisfecho crea estar el varón en su casa por disposición de un dios o por causa de un hombre, ella encuentra un motivo de reproche y se arma para la batalla. Porque donde hay una mujer, ni siquiera querrían recibir con amistad a un huésped que llega; precisamente la que parece ser más sensata, es la que mayores ultrajes infiere: pues cuando el marido está libre de toda sospecha . . . y los vecinos se divierten con él viendo cómo se equivoca. Cada uno alabará a su mujer cuando habla de ella y criticará a la de otro: ¡y no nos damos cuenta de que nos ha correspondido un lote igual! Pues Zeus ha creado esta calamidad superior a

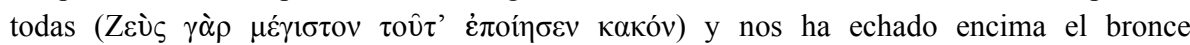
irrompible de unos grillos desde aquel día en que a unos los recibió en su mansión Hades cuando luchaban por una mujer ...»" .

El propio Arquíloco no es precisamente delicado con algunas mujeres: recordemos, sin ir más lejos, la retahíla de "piropos" que dedica a una de ellas en el fragmento 184 Bergk (= 206,

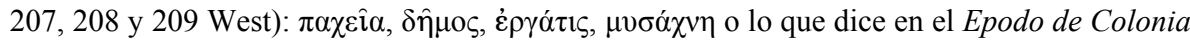
en relación con Neobula. Precisamente el Epodo de Colonia, que hemos transcrito antes parcialmente, nos da una clave interpretativa extraordinaria para el poema que nos interesa: parece ser que en ambos casos se da el mismo topos: el rechazo de una mujer por diversos motivos (por ser vieja, poco atractiva o mala persona) y la dedicación a otra más interesante. Este motivo, aunque aparece expresado de forma muy clara en el citado Epodo, se podría rastrear igualmente en otros poemas arquiloqueos, especialmente si lo consideramos en su versión más general, como desprecio machista a la mujer (lo que Pasquali ${ }^{33}$, refiriéndose a Horacio, llama "canzone a dispetto"): en el fragmento 23 West 1s. 16-21, por ejemplo, Mírmex sería felicitado por haber conseguido una mujer que no quería nadie (oủ]

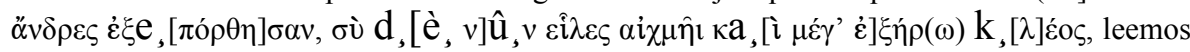
en los versos $\left.18-19^{34}\right)$. Igualmente, la literatura latina tampoco es extraña a esta idea, y así en

i corpi, gli alimenti e i fatti della natura finiscono col formare un tutto indifferenziato».

30 Cf. por ejemplo el mito de Pandora en Hes. Op. 59-106

$31 \quad$ En el fr. 7 West $=7$ Pellizer - Tedeschi.

32 Vv. 103-118. En Líricos Griegos. Elegíacos y yambógrafos arcaicos I, Madrid, 1990, pp. 160-161.

33 G. Pasquali, Orazio lirico. Studi, Firenze, 1920 (reimpr. 1966), p. 448.

34 Sobre este poema y una nueva división del papiro en que aparece, cf. mi 
Horacio (que tanto imitó a Arquíloco ${ }^{35}$ ) leemos en un desagradabilísimo poema (epodo VIII): rogare longo putidam te saeculo / uiris quid eneruet meas, / cum sit tibi dens ater et rugis uetus / frontem senectus exaret, / hietque turpis inter aridas natis / podex uelut crudae bouis? / sed incitat me pectus et mammae putres, / equina quales ubera, / uenterque mollis et femur tumentibus / exile suris additum. / esto beata, funus atque imagines / ducant triumphales tuum, / nec sit marita, quae rotundioribus / onusta bacis ambulet. / quid quid libelli Stoici inter sericos / iacere puluillos amant? / illiterati num minus nerui rigent, / minusue languet fascinum? / quod ut superbo prouoces ab inguine, / ore allaborandum est tibi ${ }^{36}$.

c) "Sayo": Ya hemos visto anteriormente que, para nosotros, "sayo" equivaldría a "borracho". Desde esta perspectiva, la indirecta de Arquíloco sería clara: otro poseerá a la mujer despreciada, pero para poseer a esta "víbora", deberá estar borracho. De otro modo, difícilmente la podría aguantar.

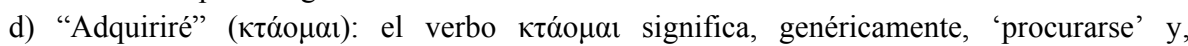
significativamente, se emplea tanto para el campo armamentístico ${ }^{37}$ como refiriéndose al acto

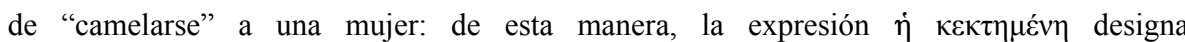
despectivamente a la mujer, como se ve claramente en los siguientes versos (61-76) de la

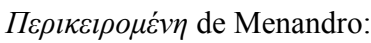

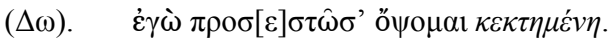

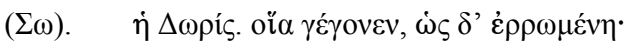

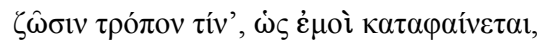

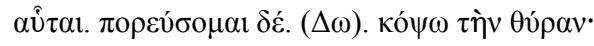

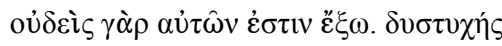

comunicación «Dues consolationes ad pathicos d'Arquíloc», presentada en el XII Congreso de la Secció Catalana de la Societat Espanyola d'Estudis Clàssics (Palma de Mallorca, 1 a 4 de febrero de 1996), Palma de Mallorca, 1997, pp. 189-194.

35 Sobre esta cuestión (ya tratada por F. Leo en su De Horatio et Archilocho, Gött. Programm, 1900, y continuada por E. Wistrand en «Archilochus and Horace», Archiloque. Entretiens sur l'Antiquité Classique de la Fondation Hard, tome X, Vandœuvres-Genève, 1963, pp. 257-287), véase recientemente G. F. Gianotti - A. Pennacini, Storia e forme della letteratura in Roma antica, Torino, 1982, p. 253: «Mentre i neoteroi avevano guardato alla poesia alessandrina come a punto di riferimento privilegiato per i propi esercizi lirici riservati agli 'addetti ai lavori', Orazio si distacca dal neoterismo perché risale oltre gli alessandrini e vuole rievocare le cadenze della lirica greca arcaica. Chi si presenta nelle vesti di un Archiloco romano ambisce farsi interlocutore di un pubblico ben più vasto di quello, seleziontissimo, che costituiva l'area di ascolto dei neoteroi: non è un caso che nei componimenti più antichi Orazio si rivolga a tutta la comunità cittadina».

36 Otras «canzone a dispetto» horacianas son Carm. I 25 y Carm. IV 13. M. G. Bonanno (L'allusione necessaria. Ricerche intertestuali sulla poesia greca e latina, Roma, 1990, pp. 85-101) ha puesto de relieve las influencias arquiloqueas sobre estas composiciones.

37 Aparece aplicado a ö $\pi \lambda \alpha$ en Hdt. I 155 y S. Ph. 778. 


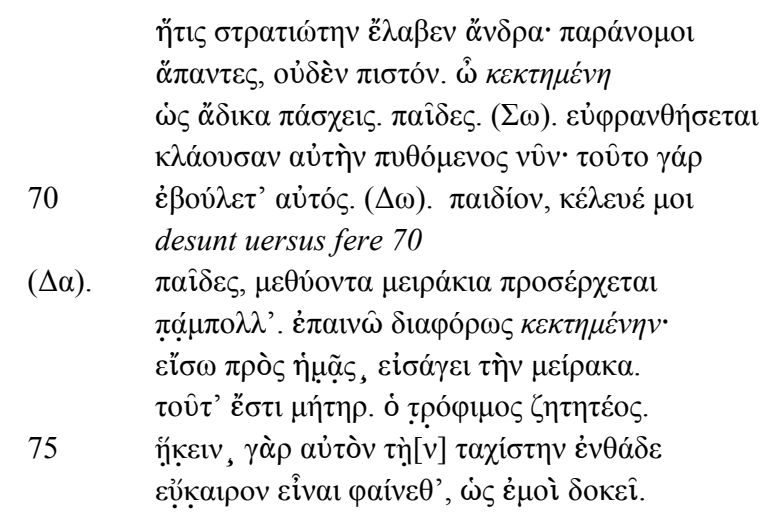

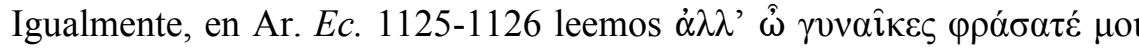

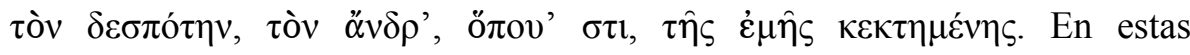
circunstancias, parece interesante el juego de palabras de Arquíloco: literalmente, adquirirá otra arma; metafóricamente, se "ligará" a otra mujer. En definitiva, como decíamos al principio de este artículo, para nosotros el fragmento 5 West admite diversas lecturas, todas ellas válidas. Este juego con la dilogía es muy propio de Arquíloco y es lo que ha motivado, a nuestro entender, que interpretaciones más atrevidas como la nuestra hayan pasado desapercibidas hasta ahora. Sin embargo, hay una serie de indicios que nos hacen ir más lejos de la interpretación meramente literal (entre ellos, tendríamos que tener en cuenta otro referido al marco de la ejecución del poema, el simposio meramente masculino ${ }^{38}$ ) y que son los que aquí hemos

38 Que este poema tuvo su ejecución en un marco simpótico es aceptado actualmente por la mayoría de críticos. Adrados, por ejemplo, escribe al respecto (en J. A. López Férez, ed., Historia de la literatura griega, Madrid, 1988, p. 126) que «también podemos pensar en el banquete como lugar de ejecución del fragmento del escudo (12), aquel que Arquíloco no lamenta porque salvó la vida y ya se comprará otro mejor». Pues bien, como señala E. Pellizer («Outlines of a Morphology of Sympotic Entertainment», in O. Murray, Sympotica. A symposium on the Symposion, Oxford, 1994, pp. 178-180) el marco del simposio es, esencialmente, el del abuso etílico y el del amor: «First of all, in the symposion are evolved those ritual norms intended to regulate the use of alcoholic drink, in particular wine, that is to say the rules of an ellaborate ars bibendi. Placed under the divine sanction of Dionysos, this art has the funcion of striking a balance, through moderation (metron) in drinking, between the extremes of complete abstinence (nephein) and harmful drunkenness (methyesthai, paroinein, kraipalan), between the tedious and bland behaviour of the sober man and the irrational and violent behaviour of the drunkard. [ . . ] A third fundamental element in the 
tratado de interpretar. Añadamos, finalmente, que si se acepta esta interpretación tendríamos una prueba más de la genialidad del poeta de Paros, un autor que es capaz de jugar simultáneamente y con toda naturalidad con dos o tres niveles de interpretación*.

symposion, about which much remains to be said, is that of the amorous discourse which is developed in the course of it. Eros and the pleasures of love figure amongst the most characteristic subjects of the logos sympotikos, both in its poetic expression and in the eloquent philosophical discussion which was to typify the 'literary' symposion from Plato and Xenophon onwards».

Agradezco al Prof. Ezio Pellizer, de la Universidad de Trieste, sus valiosos comentarios durante la confección de este artículo, así como su actitud abierta al diálogo, a pesar de sus reticencias sobre las conclusiones del trabajo. 